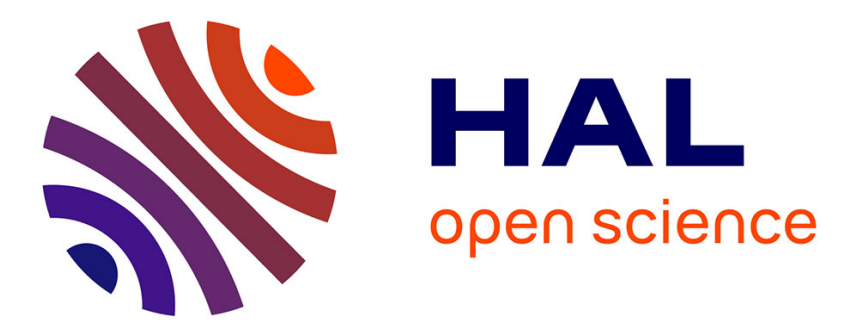

\title{
Physical Layer Security Game: How to Date a Girl with Her Boyfriend on the Same Table
}

Han Zhu, Marina Ninoslav, Merouane Debbah, Are Hjorungnes

\section{To cite this version:}

Han Zhu, Marina Ninoslav, Merouane Debbah, Are Hjorungnes. Physical Layer Security Game: How to Date a Girl with Her Boyfriend on the Same Table. IEEE International Conference on Game Theory for Networks, May 2009, Istanbul, Turkey. 8 p. hal-00392362

\section{HAL Id: hal-00392362 \\ https://hal-centralesupelec.archives-ouvertes.fr/hal-00392362}

Submitted on 7 Jun 2009

HAL is a multi-disciplinary open access archive for the deposit and dissemination of scientific research documents, whether they are published or not. The documents may come from teaching and research institutions in France or abroad, or from public or private research centers.
L'archive ouverte pluridisciplinaire HAL, est destinée au dépôt et à la diffusion de documents scientifiques de niveau recherche, publiés ou non, émanant des établissements d'enseignement et de recherche français ou étrangers, des laboratoires publics ou privés. 


\title{
Physical Layer Security Game: How to Date a Girl with Her Boyfriend on the Same Table
}

\author{
Zhu Han ${ }^{1}$, Ninoslav Marina ${ }^{2}$, Mérouane Debbah $^{3}$, and Are Hjørungnes ${ }^{2}$ \\ 1 Electrical and Computer Engineering Department, University of Houston, USA. \\ ${ }^{2}$ UniK - University Graduate Center, University of Oslo, Norway. \\ 3 Alcatel-Lucent Chair on Flexible Radio, SUPÉLEC, Gif-sur-Yvette, France.
}

\begin{abstract}
Physical layer security is an emerging security technology that achieves perfect secrecy data transmission between the intended network nodes, while the eavesdropping malicious nodes obtain zero information. The so-called secrecy capacity can be improved using friendly jammers that introduce extra interference to the eavesdropping malicious nodes while the interference to the intended destination is limited. In this paper, we investigate the interaction between the source that transmits the desired data and friendly jammers who assist the source by "disguising" the eavesdropper. In order to obtain a distributed solution, we introduce a game theoretic approach. The game is defined in such a way that the source pays the friendly jammers to interfere the eavesdropper, therefore increasing its secrecy capacit; and the friendly jammers charge the source with a certain price for the jamming. There is a tradeoff for the price: If the price is too low, the profit of the jammers is low; and if the price is too high, the source would not buy the "service" (jamming power) or would buy it from other jammers. To analyze the game outcome, we define and investigate a Stackelburg game and construct a distributed algorithm. Our analysis and simulation results show the effectiveness of friendly jamming and the tradeoff for setting the price. The fancy title comes from the fact that it is similar to a scenario where the main character, namely the "source" tries to send a dating message to a lady (the intended destination), whose poor boyfriend plays the role of the eavesdropper that may hear the message. Friends of the source, the so called "friendly jammers," try to distract the boyfriend, so that the dating message can be secretly transmitted. The game is defined in order to derive what is the optimal price that the friends can charge for this "friendly" action.

Keywords-Physical Layer Security, Secrecy Capacity,
\end{abstract} Jamming, Game Theory, and Stackelburg game

\section{INTRODUCTION}

Nino, rephrase most of sentences and add most recent literature for phy sec.

The future communication systems will be decentralized and ad-hoc, therefore allowing various types of network mobile terminals to join and leave. This aspect makes the whole system vulnerable and susceptible to attacks. Anyone within communication range can listen and possibly extract information. While these days we have numerous cryptographic methods with high level security, there is no system with perfect security on physical layer. Therefore, the physical (PHY) layer security is regaining a new attention. The main goal of this paper is to design a decentralized system that will protect the broadcasted data and

This work was supported by NSF CNS-0910461, and was supported by the Research Council of Norway through the project entitled "Mobile-to-Mobile Communication Systems (M2M)". make it impossible for the eavesdropper to receive the packets even if it knows the encoding/decoding schemes used by the transmitter/receiver. In approaches where PHY layer security is applied, the main objective is to maximize the rate of reliable information from the source to the intended destination, while all malicious nodes are kept as ignorant of that information as possible. This maximum reliable rate is known as secrecy capacity.

This line of work was pioneered by Aaron Wyner, who defined the wiretap channel and established the possibility to create almost perfect secure communication links without relying on private (secret) keys [1]. Wyner showed that when the eavesdropper channel is a degraded version of the main channel, the source and the destination can exchange perfectly secure messages at a non-zero rate. The main idea proposed by him is to exploit the additive noise impairing the eavesdropper by using a stochastic encoder that maps each message to many codewords according to an appropriate probability distribution. With this scheme, a maximal equivocation (i.e., uncertainty) is induced at the eavesdropper. In other words, a maximal level of secrecy is obtained. By ensuring that the equivocation rate is arbitrarily close to the message rate, one can achieve perfect secrecy in the sense that the eavesdropper is now limited to learn almost nothing about the source-destination messages from its observations. Follow-up work by Leung-YanCheong and Hellman characterized the secrecy capacity of the additive white Gaussian noise (AWGN) wiretap channel [2]. In their landmark paper, Csiszár and Körner generalized Wyner's approach by considering the transmission of confidential messages over broadcast channels [3]. Recently, there have been considerable efforts on generalizing these studies to the wireless channel and multi-user scenarios (see [4-12] and references therein). Jamming [13-15] has been studied for a long time to analyze the hostile behaviors of malicious nodes. Recently, jamming has been employed to physical layer security to reduce the eavesdropper's ability to decode the source's information [16]. In other words, the jamming is friendly in this context.

Game theory [17] is a formal framework with a set of mathematical tools to study the complex interactions among interdependent rational players. For more than half a century, game theory has led to revolutionary changes in economics, and has found important applications in politics, sociology, psychology, transportation etc. During the past decade, there has been a surge in research activities 
that employ game theory to model and analyze modern communication systems. This is mainly due to: (1) the emergence of the Internet as a global platform for computation and communication, which has sparked the development of large-scale, distributed and heterogeneous communication systems; (2) the deregulation of the telecommunication industry and the dramatic improvement in computation power, which make it possible for various network entities to make independent and selfish operational decisions; and (3) the need for robust designs against uncertainties modeled as games between the user and a malicious nature. Most of these works [18-21] concentrate on the distributed resource allocation for wireless networks. As far as the authors' knowledge, the game theory has not yet been used in the physical layer security.

In this paper, we investigate the interaction between the source and its friendly jammers using game theory. Although the friendly jammers help the source by reducing the data rate that is "leaking" from the source to the malicious node, at the same time they also reduce the useful data rate from the source to the destination. Using well chosen amounts of power from the friendly jammers, the secrecy capacity can be maximized. In the game that we define here, the source pays the jammers to interfere the malicious eavesdropper, and therefore, to increase the secrecy capacity. The friendly jammers charge the source with a certain price for the jamming the eavesdropper. One could notice that there is a tradeoff for the proposed price: If the price of a certain jammer is too low, its profit is also low; if its price is too high, the source will buy from the other jammers. In modeling the outcome of the above games our analysis uses the Stackelberg type of game. Initially, the existence of equilibrium will be studied. Then, a distributed algorithm will be proposed and its convergence will be investigated. The outcome of the distributed algorithm will be compared to the centralized genie aided solution. Some implementation concerns are also discussed. From the simulation results, we can see the efficiency of friendly jamming and tradeoff for setting the price, the source prefers buying service from only one jammer, and the centralized scheme and the proposed game scheme has similar performance.

As for the fancy title, the source is the main character, the destination is a lovely girl, and her poor boyfriend resembles the eavesdropper. A friend (jammer) or several friends distract the boyfriend, so that the dating message can be secretly transmitted from the main character (the source) to the girl (the destination). The game is defined as follows: How much these friends should charge the main character for this "friendly" action. The authors hope the fancy title can attract more attentions on this research track for distributed solution of physical layer security problems.

The rest of the paper is organized as follows: In Section II, the system model of physical layer security with friendly jamming users is described. In Section III, the game models are formulated, and the outcomes as well as properties of the game are analyzed. Simulation results are shown in
Fig. 1. System Model for Physical Layer Security Game

Section IV and conclusions are drawn in Section V.

\section{SyStem MOdeL}

We consider a network with a source, a destination, a malicious eavesdropper node, and $J$ friendly jammer nodes as shown in Figure $1^{1}$. The malicious node tries to eavesdrop the transmitted data coming from the source node. When the eavesdropper channel from the source to the malicious node is a degraded version of the main source-destination channel, the source and destination can exchange perfectly secure messages at a non-zero rate. By transmitting a message at a rate higher than the rate of the malicious node, the malicious node can learn almost nothing about the messages from its observations. The maximum rate of secrecy information from the source to its intended destination is defined by the term secrecy capacity.

Suppose the source transmits with power $P_{0}$. The channel gains from the source to the destination and from the source to the malicious node are $G_{s d}$ and $G_{s m}$, respectively. Each friendly jammer $i, i=1, \ldots, J$ transmits with power $P_{i}$ and the channel gains from it to the destination and the malicious node, are $G_{i d}$ and $G_{i m}$, respectively. For convenience, we denote by $\mathcal{J}$ the set of indices $\{1,2, \ldots, J\}$. If the path loss model is used, the channel gain is given by the distance to the negative power of the path loss coefficient. The thermal noise for each channel is $\sigma^{2}$ and the bandwidth is $W$. The channel capacity for the source to the destination is

$$
C_{1}=W \log _{2}\left(1+\frac{P_{0} G_{s d}}{\sigma^{2}+\sum_{i \in \mathcal{J}} P_{i} G_{i d}}\right)
$$

The channel capacity from the source to the malicious node is

$$
C_{2}=W \log _{2}\left(1+\frac{P_{0} G_{s m}}{\sigma^{2}+\sum_{i \in \mathcal{J}} P_{i} G_{i m}}\right) .
$$

In order to ensure that the eavesdropping malicious node can obtain zero mutual information from the source, the source should send its data with the secrecy capacity as

$$
C_{s}=\left(C_{1}-C_{2}\right)^{+}
$$

\footnotetext{
${ }^{1}$ Multiple source-destination pairs and multiple malicious nodes case will be considered for our future work.
} 
where $(\cdot)^{+}=\max (\cdot, 0)$. We can see that with the increase of the jamming power $P_{i}$, both $C_{1}$ and $C_{2}$ are reduced. The questions are whether or not $C_{s}$ can be increased, and how to control the jamming power in a distributed manner. We will try to solve the problems in the following section using a game theoretical approach.

It is worth mentioning that the system model used in this paper is additive white Gaussian noise (AWGN) channel, which can provide some insights on the game and interactions between source and the friendly jammers. For more sophisticated scenarios such as Rayleigh fading, it is usually assumed that the source-destination channels are known but only the channel statistics of source-jamming channel are known. The problem is how to write (3), while the rest of derivations of this paper can be performed in a similar way.

\section{Game for Physical Layer Security}

In this section, we study how to use game theory to analyze the physical layer security. First, we define the game between the source and friendly jammers. Next, we optimize the source and jammer sides, respectively. Then, we prove some properties of the proposed game. Finally, we discuss some implementation concerns.

\section{A. Game Definition}

The source can be modeled as a buyer who wants to optimize its secrecy capacity minus cost by modifying the "service" (jamming power $P_{i}$ ) from the friendly jammers, i.e.,

$$
\begin{gathered}
\text { Source's Game: } \max U_{s}=\left(a \max \left(C_{1}-C_{2}, 0\right)-M\right), \\
\text { s.t. } P_{i} \leq P_{\max },
\end{gathered}
$$

where $a$ is the gain per unit capacity, $P_{\max }$ is the maximal power that a jammer can provide, and $M$ is the cost to pay for the other friendly jamming nodes. Here

$$
M=\sum_{i \in \mathcal{J}} p_{i} P_{i},
$$

where $p_{i}$ is the price per unit power for the friendly jammer, $P_{i}$ is the friendly jammer's power, and $\mathcal{J}$ is the set of friendly jammers. From (4) we note that the source will not participate in the game if $C_{1}<C_{2}$, or in other words, the secrecy capacity is zero. For each jammer, $U_{i}\left(p_{i}, P_{i}\left(p_{i}\right)\right)$, is the utility function of the price and power bought by the source. For the jammer's (seller's) utility, in this paper we define the following utility

$$
U_{i}=p_{i} P_{i}^{c_{i}},
$$

where $c_{i} \geq 1$ is a constant to balance from the payment $p_{i} P_{i}$ from the source and the transmission cost $P_{i}$. Notice that $P_{i}$ is also a function of the vector of prices $\left(p_{1}, \ldots p_{N}\right)$ since the power that the source will buy also depends on the price that the friendly jammers ask. Hence, for each friendly jammer, the optimization problem is

$$
\text { Friendly Jammer's Game: } \max _{p_{i}} U_{i} \text {. }
$$

The games for the source and friendly jammer are similar to the games between buyers and sellers. In the next two subsections, we analyze the optimal strategies for the source and friendly jammers to maximize their own utilities. The analysis is similar to Stackelburg game in the literature [17].

\section{B. Source (Buyer) Side Analysis}

The goal of the source as a buyer is to buy the optimal amount of the power from the friendly jammers so as to improve its secrecy capacity. We introducing the following definitions:

$$
\begin{gathered}
A=P_{0} G_{s d} / \sigma^{2}, \\
B=P_{0} G_{s m} / \sigma^{2}, \\
u_{i}=G_{i d} / \sigma^{2},
\end{gathered}
$$

and

$$
v_{i}=G_{i m} / \sigma^{2}, i \in \mathcal{J} .
$$

From (4), we have

$$
\begin{array}{r}
U_{s}=a W\left(\log \left(1+\frac{A}{1+\sum_{j \in \mathcal{J}} u_{j} P_{j}}\right)\right. \\
\left.-\log \left(1+\frac{B}{1+\sum_{j \in \mathcal{J}} v_{j} P_{j}}\right)\right)^{+}-\sum_{j \in \mathcal{J}} p_{j} P_{j},
\end{array}
$$

For the source (buyer) size, we first analyze the case where $C_{1}>C_{2}$, i.e., the secrecy capacity is not zero before the friendly jammers' participation. By differentiating (4), we have

$$
\begin{array}{r}
\frac{\partial U_{s}}{\partial P_{i}}=-\frac{a W A u_{i} / \ln 2}{\left(1+A+\sum_{j \in \mathcal{J}} u_{j} P_{j}\right)\left(1+\sum_{j \in \mathcal{J}} u_{j} P_{j}\right)} \\
+\frac{a W B v_{i} / \ln 2}{\left(1+B+\sum_{j \in \mathcal{J}} v_{j} P_{j}\right)\left(1+\sum_{j \in \mathcal{J}} v_{j} P_{j}\right)}-p_{i}=0 .
\end{array}
$$

Rearranging the above equation, we have

$P_{i}^{4}+F_{i, 3} P_{i}^{3}+F_{i, 2}\left(p_{i}\right) P_{i}^{2}+F_{i, 1}\left(p_{i}\right) P_{i}+F_{i, 0}\left(p_{i}\right)=0$,

where

$$
\begin{aligned}
& F_{i, 3}=\left(2+2 \alpha_{i}+A\right)^{2}+\left(2+2 \beta_{i}+B\right)^{2} \\
& F_{i, 2}\left(p_{i}\right)=\frac{\left(2+2 \alpha_{i}+A\right)\left(2+2 \beta_{i}+B\right)}{u_{i} v_{i}} \\
& +\frac{L_{i}}{v_{i}^{2}}+\frac{K_{i}}{u_{i}^{2}}-\frac{a W}{p_{i} u_{i} v_{i}}\left(\frac{B}{v_{i}}-\frac{A}{u_{i}}\right) \\
& F_{i, 1}\left(p_{i}\right)=\frac{L_{i} C_{i}+K_{i} D_{i}}{u_{i}^{2} v_{i}^{2}}+\frac{a W\left(A D_{i}-B C_{i}\right)}{p_{i} u_{i}^{2} v_{i}^{2}} \\
& F_{i, 0}\left(p_{i}\right)=\frac{K_{i} L_{i}}{u_{i}^{2} v_{i}^{2}}+\frac{a W\left(A u_{i} L_{i}-B v_{i} K_{i}\right)}{p_{i} u_{i}^{2} v_{i}^{2}}
\end{aligned}
$$


and

$$
\begin{gathered}
\alpha_{i}=\sum_{j \neq i} G_{j d} P_{j}, \\
\beta_{i}=\sum_{j \neq i} G_{j m} P_{j}, \\
K_{i}=\left(1+\alpha_{i}\right)\left(1+\alpha_{i}+A\right), \\
L_{i}=\left(1+\beta_{i}\right)\left(1+\beta_{i}+B\right), \\
C_{i}=u_{i}\left(2+2 \alpha_{i}+A\right), \\
D_{i}=v_{i}\left(2+2 \beta_{i}+B\right) .
\end{gathered}
$$

The solutions of the quartic can be expressed in closed form but this is not the primary goal here. It is important that the solution we are interested in is given by the following function

$$
P_{i}^{*}=P_{i}^{*}\left(p_{i}, A, B,\left\{u_{j}\right\},\left\{v_{j}\right\},\left\{P_{j}\right\}_{j \neq i}\right),
$$

which is a function of the friendly jammer's price $p_{i}$ and the other system parameters. Note that $0 \leq P_{i} \leq P_{\max }$. Since $P_{i}$ satisfies the polynomial function, we can have the optimal strategy as

$$
P_{i}^{*}=\min \left[\max \left(P_{i}, 0\right), P_{\max }\right] .
$$

Because of the complexity of the closed form solution of a quartic equation in (26), we also consider two special cases: lower interference case and high interference case in the following two subsubsections.

B.1 Interference at the Destination is much Smaller than the Noise

Remember the definitions: $A=P_{0} G_{s d} / \sigma^{2}, B=$ $P_{0} G_{s m} / \sigma^{2}, u_{i}=G_{i d} / \sigma^{2}$ and $v_{i}=G_{i m} / \sigma^{2}$. Imagine a situation in which all jammers are close to the malicious node and far from the destination node. In that case the interference from the jammers to the destination is very small in comparison to the additive noise. Therefore by omitting interfering terms, we have

$$
\begin{array}{r}
U_{s} \approx a W\left(\log (1+A)-\log \left(1+\frac{B}{1+\sum_{j \in \mathcal{J}} v_{j} P_{j}}\right)\right)^{+} \\
-\sum_{j \in \mathcal{J}} p_{j} P_{j} .
\end{array}
$$

Then by differentiating over the power the source would like to buy and setting the result to zero, we have

$$
\frac{\partial U_{s}}{\partial P_{i}}=\frac{a W B v_{i} / \ln 2}{\left(1+B+\sum_{j \in \mathcal{J}} v_{j} P_{j}\right)\left(1+\sum_{j \in \mathcal{J}} v_{j} P_{j}\right)}-p_{i}=0
$$

Rearranging the above equation, we get a polynomial function

$$
\begin{array}{r}
P_{i}^{2}+\frac{2+2 \beta_{i}+B}{v_{i}} P_{i}+\frac{\left(1+\beta_{i}\right)\left(1+B+\beta_{i}\right)}{v_{i}^{2}} \\
-\frac{a W B}{p_{i} v_{i} \ln 2}=0 .
\end{array}
$$

Solving the above equation we obtain a closed-form solution

$$
\begin{array}{r}
P_{i}^{*}=-\frac{2+2 \beta_{i}+B}{2 v_{i}}+ \\
\sqrt{\frac{\left(2+2 \beta_{i}+B\right)^{2}}{4 v_{i}^{2}}-\frac{\left(1+\beta_{i}\right)\left(1+B+\beta_{i}\right)}{v_{i}^{2}}+\frac{a W B}{p_{i} v_{i} \ln 2}} \\
=q_{i}+\sqrt{w_{i}+\frac{z_{i}}{p_{i}}} .
\end{array}
$$

Finally, by comparing $P_{i}^{*}$ with the power under the boundary conditions $\left(P_{i}=0, P_{i}=P_{\max }\right.$ and $\left.C_{s}=0\right)$, the optimal $P_{i}^{*}$ in the low SNR region can be obtained.

B.2 One Jammer with Interference that is much Higher than the Noise but much Smaller than the Received Power at the Destination and the Malicious Node

In this special case, the interference from the jammer is much higher than the additive noise but much smaller than the power of the received signal at the destination and the malicious node. In other words, that means $1<<u_{1} P_{1}<<$ $A$ and $1<<v_{1} P_{1}<<B$. Therefore, the utility function of the source is given by

$$
\begin{aligned}
U_{s} \approx a W\left(\log \left(1+\frac{A}{u_{1} P_{1}}\right)-\right. & \left.\log \left(1+\frac{B}{v_{1} P_{1}}\right)\right)-p_{1} P_{1} \\
& \approx \frac{a W A}{u_{1} P_{1}}-\frac{a W B}{v_{1} P_{1}}-p_{1} P_{1}
\end{aligned}
$$

In order to find the optimal power to buy, similarly we calculate

$$
\frac{\partial U_{s}}{\partial P_{i}}=-\frac{a W A}{u_{1} P_{1}^{2}}+\frac{a W B}{v_{1} P_{1}^{2}}-p_{1}=0 .
$$

Hence

$$
P_{1}^{*}=\sqrt{\frac{a W}{p_{1}}\left(\frac{B}{v_{1}}-\frac{A}{u_{1}}\right)}=\sqrt{\frac{D_{1}}{p_{1}}} .
$$

From this equation we get the optimal closed-form solution $P_{i}^{*}$, and similarly by comparing $P_{1}^{*}$ with the power under the boundary conditions $\left(P_{1}=0, P_{1}=P_{\max }\right.$ and $\left.C_{s}=0\right)$, we can obtain the optimal solution of the source for the this special case.

\section{Friendly Jammer (Seller) Side Analysis}

From (25), we can see that the power that a source would buy is related to the prices that the friendly jammers set. In this subsection, we study how the friendly jammers can set the optimal price to maximize its utility. By differentiating the friendly jammer's utility in (6) and setting it to zero, we have

$$
\frac{\partial U_{i}}{\partial p_{i}}=\left(P_{i}^{*}\right)^{c_{i}}+p_{i} c_{i}\left(P_{i}^{*}\right)^{c_{i}-1} \frac{\partial P_{i}^{*}}{\partial p_{i}}=0 .
$$

This is equivalent to

$$
\left(P_{i}^{*}\right)^{c_{i}-1}\left(P_{i}^{*}+p_{i} c_{i} \cdot \frac{\partial P_{i}^{*}}{\partial p_{i}}\right)=0 .
$$


This happens either if $P_{i}^{*}=0$ (the source does not buy anything from the friendly jammer) or if

$$
P_{i}^{*}+p_{i} c_{i} \cdot \frac{\partial P_{i}^{*}}{\partial p_{i}}=0 .
$$

From the closed form solution of $P_{i}^{*}$, the solution of $p_{i}^{*}$ will be a function given as

$$
p_{i}^{*}=p_{i}^{*}\left(\sigma^{2}, G_{s d}, G_{s m},\left\{G_{i d}\right\},\left\{G_{i m}\right\}\right) .
$$

Notice that $p_{i}^{*}$ should be positive. Otherwise, the friendly jammer would not play.

\section{Properties}

In this subsection, we prove some properties of the proposed game. First, we prove that the power is monotonous function of the price under the two extreme cases. The properties can help for the proof of equilibrium existence in the later part of this subsection.

Property 1: Under the two special cases, the optimal power consumption $P_{i}^{*}$ for friendly jammer $i$ is monotonous with its price $p_{i}$, when the other friendly jammers prices are fixed. The proof is straightforward from (31) and (35).

We investigate the following analysis of the relation between the price and power. We find out that the friendly jammer power $P_{i}$ bought from the source is convex in its own price $p_{i}$ under some conditions. To prove this we need to check whether the second derivative $\partial^{2} P_{i} / \partial p_{i}^{2}<0$.

In the first special case in which the interference is small, we have the first order derivative as

$$
\frac{\partial P_{i}^{*}}{\partial p_{i}}=-\frac{z_{i}}{2 p_{i}^{2} \sqrt{w_{i}+\frac{z_{i}}{p_{i}}}}
$$

and the second order derivative as

$$
\frac{\partial^{2} P_{i}^{*}}{\partial p_{i}^{2}}=\frac{z_{i}}{p_{i}^{3}\left(w_{i}+\frac{z_{i}}{p_{i}}\right)^{1 / 2}}\left(1-\frac{1}{4\left(\frac{p_{i} w_{i}}{z_{i}}+1\right)}\right) .
$$

The above equation is greater than zero when $p_{i}$ is small. This means when the interference is small and the price is small, the power is convex as a function of the price.

In the second special case in which the interference is severe, we have the first order derivative

$$
\frac{\partial P_{i}^{*}}{\partial p_{i}}=-\frac{1}{2} \sqrt{D_{1}} p_{1}^{-3 / 2}
$$

and the second order derivative as

$$
\frac{\partial^{2} P_{i}^{*}}{\partial p_{i}^{2}}=\frac{3}{4} \sqrt{D_{1}} p_{1}^{-5 / 2}>0 .
$$

This means when the interference is severe, the power is a convex function of the price.

Next, we investigate the equilibrium of the proposed game. In other word, no user can improve the its utility by changing its own strategy only. We first define the Stackelberg equilibrium as follow:
Definition 1: $P_{i}^{S E}$ and $p_{i}^{S E}$ are the Stackelberg equilibrium of the proposed game, if when $p_{i}$ is fixed,

$$
U_{s}\left(\left\{P_{i}^{S E}\right\}\right)=\sup _{P_{\max } \geq\left\{P_{i}^{S E}\right\} \geq 0, \forall i} U_{s}\left(\left\{P_{i}\right\}\right), \forall i \in \mathcal{J}
$$

and when $P_{i}$ is fixed,

$$
U_{i}\left(p_{i}^{S E}\right)=\sup _{p_{i}} U_{i}\left(p_{i}\right), \forall i \in \mathcal{J} .
$$

Finally, from the analysis in the previous two subsections, we can shown the following property for the proposed game.

Property 2: The pair of $\left\{P_{i}^{*}\right\}_{i=1}^{N}$ in (26) and $\left\{p_{i}^{*}\right\}_{i=1}^{N}$ in (39) is the Stackelberg equilibrium for the proposed game.

\section{E. Distributed Algorithm and Convergence}

In this subsection, we study how the distributed game can converge to the Stackelberg equilibrium defined in the above subsection. After rearranging (36), we have

$$
p_{i}=I_{i}(\mathbf{p})=-\frac{\left(P_{i}^{*}\right)}{c_{i} \frac{\partial P_{i}^{*}}{\partial p_{i}}},
$$

where price vector $\mathbf{p}=\left[p_{1}, \ldots, p_{N}\right]^{T}$ and $I_{i}(\mathbf{p})$ is the price update function. Notice that the optimal power $P_{i}^{*}$ is a function of price vector $\mathbf{p}$. The information for the update can be obtained from the source node. This is similar to the distributed power control [24]. The update of the friendly jammers' prices can be written in a vector form as

$$
\text { Distributed Algorithm: } \mathbf{p}(t+1)=\mathbf{I}(\mathbf{p}(t)) \text {, }
$$

where $\mathbf{I}=\left[I_{1}, \ldots, I_{N}\right]^{T}$, and the iteration is from time $t$ to time $t+1$. Next, we show that the convergence of the proposed scheme using the update in (47) by proving that the price update function in (47) is a standard function [22] defined as

Definition 2: A function $\mathbf{I}(\mathbf{p})$ is standard, if for all $\mathbf{p} \geq$ $\mathbf{0}$, the following properties are satisfied

1. Positivity: $\mathbf{p}>\mathbf{0}$,

2. Monotonicity: if $\mathbf{p} \geq \mathbf{p}^{\prime}$, then $\mathbf{I}(\mathbf{p}) \geq \mathbf{I}\left(\mathbf{p}^{\prime}\right)$, or $\mathbf{I}(\mathbf{p}) \leq$ $\mathbf{I}\left(\mathbf{p}^{\prime}\right)$,

3. Scalability: For all $\eta>1, \eta \mathbf{I}(\mathbf{p}) \geq \mathbf{I}(\eta \mathbf{p})$.

In [22], it has been proved that the price will converge to the fixed point (i.e. the Stackelberg equilibrium in our case) from any feasible initial price vector.

The positivity is very easy to prove. If the price $p_{i}$ increases, the source would buy less from the $i^{\text {th }}$ friendly jammer. As a result, $\frac{\partial P_{i}^{*}}{\partial p_{i}}$ in $(36)$ is negative, and we prove positivity $p_{i}=I_{i}(\mathbf{p})>0$.

For monotonicity and scalability, we can only show the two special cases. For the low interference case, from (31) it is obvious that

$$
\begin{aligned}
I_{i}(\mathbf{p}) & =-\frac{\left(P_{i}^{*}\right)}{c_{i} \frac{\partial P_{i}^{*}}{\partial p_{i}}} \\
& =\frac{2 \sqrt{w_{i} p_{i}^{2}+z_{i} p_{i}}\left(q_{i} p_{i}+\sqrt{w_{i} p_{i}^{2}+z_{i} p_{i}}\right)}{c_{i} z_{i}}
\end{aligned}
$$


Fig. 2. Secrecy Capacity vs. Jamming Power

which is monotonically increasing in $p_{i}$. For scalability, we have

$$
\frac{I_{i}(\eta \mathbf{p})}{\eta I_{i}(\mathbf{p})}=\frac{\sqrt{w_{i} p_{i}^{2}+z_{i} p_{i} / \eta}\left(q_{i} p_{i}+\sqrt{w_{i} p_{i}^{2}+z_{i} p_{i} / \eta}\right)}{\sqrt{w_{i} p_{i}^{2}+z_{i} p_{i}}\left(q_{i} p_{i}+\sqrt{w_{i} p_{i}^{2}+z_{i} p_{i}}\right)}<1,
$$

since $\eta>1$.

For the large interference case, from (35) we have

$$
I_{i}(\mathbf{p})=-\frac{\left(P_{i}^{*}\right)}{c_{i} \frac{\partial P_{i}^{*}}{\partial p_{i}}}=\frac{2 p_{i}}{c_{i}}
$$

which is monotonically increasing in $p_{i}$ and scalable.

Based on the above analysis, we can conclude that under the two special cases, the game will converge to the Stackelberg equilibrium from any arbitrary feasible solution. For the other cases, the proof cannot be mathematically trackable. From the observation in the simulations, the price and power indeed converge.

\section{F. Implementation Discussion}

There are several implementation concerns for the proposed scheme. First, the channel information from the source to the malicious eavesdropper might not be known or accurately known. Under this condition, the secrecy capacity formula should be rewritten considering the uncertainty. The close form solution might be hard to be found, some numerical solutions might be able to be obtained. Moreover, some side information can also be helpful. For example, if the direction of arrival is known, multiple antenna techniques can be employed such as in [12].

Second, the proposed scheme need iteratively updating the price and power information. A natural question arises that if the distributed scheme has less signalling than the centralize scheme. The comparison is similar to distributed and centralized power control in the literature [22,24]. Since the channel condition is continuously changing, the distributed solution only needs to update the difference of the parameters such as power and price to be adaptive,
Fig. 3. How much Power the Source Would Buy vs. Price

while the centralized scheme requires all channel information in each time period. As a result, the distributed solution has a clear advantage and dominates the current and future wireless network design. For example, the power control for cellular networks, the open loop power control is done only once during the link initialization, while the close loop power control (distributed power allocation such as [22]) is performed 1500 times for UMTS and 800 times for CDMA2000.

Finally, for the multi-source-destination-pair multieavesdropper case, there are two possible choices to solve the problem. First, we can use clustering method to divide the network into sub-networks, and then employ the singlesource-destination pair and multiple-friendly-jammer solution proposed in this paper. Or if we consider the jamming power can be useful for multiple eavesdroppers, some techniques such as double auction can be investigated. The detailed discussion is beyond the scope of this paper and would be considered in our future research.

\section{Simulation Results}

The simulation is set up as follows: The source and friendly jammer have power of 0.02 , the bandwidth is 1 , the noise level is $10^{-8}$, the propagation loss factor is 3 , AWGN channel is assumed. the source, destination, and eavesdropper are located at the coordinate $(0,0),(100,0)$, and $(50,50)$, respectively. Here we select $a=2$ for the friendly jammer utility in (6).

For single friendly jammer case, we show the simulation with the friendly jammer at the location of $(50,75)$ and $(10,75)$. In Figure 2, we show the secrecy capacity as a function of jamming power. We can see that with the increase of the jamming power, the secrecy capacity first increases and then decreases. This is because the jamming power has different effects on $C_{1}$ and $C_{2}$. So there is an optimal point for the jamming power. Also the optimal point depends on the location of the friendly jammer, and the friendly jammer close to the eavesdropper is more effective to improve the secrecy capacity. Moreover, notice that the 
Fig. 4. $U_{s}$ vs. Prices of Two Users

Fig. 5. $U_{1}$ vs. Prices of Two Users

curve is not convex and not concave. In Figure 3, we show the how much power the source buys from the jammer as a function of the requested price. We can see that the power is reduced if the price goes high. At some point, the source would stop buying the power. So there is a tradeoff for setting the price, i.e., if the price too high, the source would buy less power or even would buy nothing.

For the two-user case, we set up the following simulations. Malicious node is located at $(50,90)$, friendly jammer one is located at $(50,50)$, and friendly jammer two is located at $(50,75)$. In Figure 4, Figure 5, and Figure 6, we show the source's utility $U_{s}$, jammer one's utility $U_{1}$, and jammer two's utility $U_{2}$ as function of both users' price, respectively. We can see that the source would buy service from only one of the friendly jammers. If the friendly jammer asks too low price, the jammer's utility is very low. On the other hand, if the jammer asks too high price, it risks the situation in which the source would buy the service from the other friendly jammer. There is an optimal price for each friendly jammer to ask, and the source would al-
Fig. 6. $U_{2}$ vs. Prices of Two Users

ways select the one that can provide the best performance improvement.

\section{Conclusions}

Physical layer security is an emerging security technique that is an alternative for traditional cryptographic-based protocols to achieves perfect secrecy capacity as eavesdroppers obtain zero information. Jamming has been shown in the literature to effectively improve secrecy capacity. In this paper, we investigate the interaction between the source and friendly jammers using the game theory so as to have a distributed solution. The source pays the friendly jammers to interfere the malicious eavesdropper so as to increase the secrecy capacity. The friendly jammers charge the source with a price for the jamming. To analyze the game outcome, we investigate the Stackelburg game and construct the distributed algorithm. Some properties such as equilibrium and convergence are analyzed. From the simulation results, we can see the following points. First, there is a tradeoff for the price: if the price is too low, the profit is low; if the price is too high, the source would not buy or buy from the other jammers. Second, for the multiple jammer case, the source would buy service from only one jammer. Overall, the proposed game theoretical scheme can achieve a good performance with distributed implementation.

\section{REFERENCES}

[1] A. D. Wyner, "The wire-tap channel," Bell System Technical Journal, vol. 54, no. 8, pp. 1355-1387, 1975.

[2] S. K. Leung-Yan-Cheong and M. E. Hellman, "The Gaussian wiretap channel," IEEE Transactions on Information Theory, vol. 24, pp. 451 - 456, July 1978.

[3] I. Csiszar and J. Korner, "Broadcast channels with confidential messages," IEEE Transactions on Information Theory, vol. 24, pp. 339 - 348, May 1978.

[4] A. O. Hero, "Secure space-time communication," IEEE Transactions on Information Theory, vol. 49, no.12, pp. 3235 -3249, December 2003.

[5] S. K. Leung-Yan-Cheong and M. E. Hellman, "The Gaussian wiretap channel," IEEE Transactions on Information Theory, vol. 24, pp. $451-456$, July 1978. 
[6] Z. Li, W. Trappe and R. Yates, "Secret communication via multiantenna transmission," in Proc. of 41st Conference on Information Sciences and Systems, Baltimore, MD, March 2007.

[7] R. Negi and S. Goelm "Secret communication using artificial noise," in Proc. of IEEE Vehicular Technology Conference, vol. 3, pp. 1906-1910, September 2005.

[8] P. Parada and R. Blahut, "Secrecy capacity of SIMO and slow fading channels." in Proc. of IEEE International Symposium on Information Theory, pp. 2152 - 2155, September 2005.

[9] S. Shafiee and S. Ulukus, "Achievable rates in Gaussian MISO channels with secrecy constraints," in Proc. of IEEE International Symposium on Information Theory, Nice, France, June 2007.

[10] Y. Liang, H. V. Poor, and S. Shamai (Shitz), "Secure communication over fading channels", IEEE Transactions on Information Theory, vol. 54, no. 6, p.p. 2470-2492, June 2008.

[11] P. K. Gopala, L. Lai, and H. El Gamal, "On the secrecy capacity of fading channels," IEEE Transactions on Information Theory, to appear.

[12] L. Dong, Z. Han, A. P. Petropulu, and H. V. Poor, "Secure collaborative beamforming", in Proc. of Allerton Conference on Communication, Control, and Computing, Allerton, IL, October 2008.

[13] A. Kashyap, T. Basar, and R. Srikant, "Correlated jamming on MIMO Gaussian fading channels", IEEE Transactions on Information Theory, Vol. 50, Issue: 9, Page: 2119- 2123, Sept. 2004.

[14] S. Shafiee and S. Ulukus, "Mutual information games in multi-user channels with correlated jamming", http : //arxiv.org/abs/cs.IT/0601110

[15] M. H. Brady, M. Mohseni, and J. M. Cioffi, "Spatially-correlated jamming in gaussian multiple access and broadcast channels", in Proc. of 40th Annual Conference on Information Sciences and Systems, Princeton, NJ, March 2006.

[16] L. Lai and H. El Gamal, "The relay-eavesdropper channel: Cooperation for secrecy," IEEE Transactions on Information Theory, to appear,

[17] D. Fudenberg and J. Tirole, Game theory, MIT Press, Cambridge, MA, 1991.

[18] C. U. Saraydar, N. B. Mandayam, and D. J. Goodman, "Efficient power control via pricing in wireless data networks", IEEE Transations on Communications, vol.50, no.2, p.p.291-303, Feburary 2002.

[19] G. Scutari, S. Barbarossa, and D. P. Palomar, "Potential games: a framework for vector power control problems with coupled constraints", in Proc. of IEEE International Conference on Acoustics, Speech and Signal Processing, (ICASSP), Volume 4, Issue , 14-19 May 2006.

[20] B. Wang, Z. Han, and K. J. R. Liu, "Distributed relay selection and power control for multiuser cooperative communication networks using buyer / seller Game", in Proc. of Annual IEEE Conference on Computer Communications, INFOCOM, Anchorage, AK, May 2007.

[21] N. Bonneau, M. Debbah, E. Altman, and A. Hjørungnes, "Nonatomic games for multi-user systems" IEEE Journal on Selected Areas in Communications, issue on "Game Theory in Communication Systems", vol.26, no.7, p.p.1047-1058, September 2008.

[22] R. Yates, "A framework for uplink power control in cellular radio systems", IEEE Journals on Selected Areas on Commununications, vol.13, no.7, pp.1341-1348, September 1995.

[23] S. Boyd and L. Vandenberghe, Convex optimization, Cambridge University Press, 2006. (http://www.stanford.edu/ boyd/cvxbook.html)

[24] Z. Han and K. J. R. Liu, Resource Allocation for Wireless Networks: Basics, Techniques, and Applications, Cambridge University Press, UK, April, 2008. 